\title{
Understanding Nonlinear Dissolution Rates in Photoresists
}

\author{
Sean D. Burns ${ }^{\mathrm{a}}$, Allen B. Gardiner ${ }^{\mathrm{b}}$, V.J. Krukonis ${ }^{\mathrm{c}}$, Paula M. Wetmore ${ }^{\mathrm{c}}$, Jodie Lutkenhaus ${ }^{\mathrm{a}}$, Gerard M. \\ Schmid $^{\mathrm{a}}$, Lewis W. Flanagin ${ }^{\mathrm{d}}$ and C. Grant Willson ${ }^{\mathrm{a}}$ \\ ${ }^{a}$ The Department of Chemical Engineering, University of Texas at Austin, Austin TX, 78751 \\ ${ }^{b}$ Currently at Intel Corp., 5200 N.E. Elam Young Pkwy, Hillsboro OR, 97124-6497 \\ ${ }^{c}$ Phasex Corp., 360 Merrimack St., Lawrence, MA 01843 \\ ${ }^{\mathrm{d}}$ Texas Instruments Inc., 13570 N. Central Expressway, MS 3701, Dallas, TX 75243
}

\begin{abstract}
This work focuses on understanding the dissolution phenomenon of surface inhibition, which is observed often in the development of novolac based resists. Many theories have been offered to explain this phenomenon, including a concentration gradient of resist components, oxidation of the surface, formation of a gel layer, and surface roughness effects. This work focuses on theories that propose a concentration gradient in resist components. A technique has been established to separate and analyze individual layers of thin films, and the concentration gradient in many resist components (residual solvent, low molecular weight chains, photoactive compound, density) has been compared to the observed dissolution rate. The results indicate that no significant concentration gradients exist in a $1 \mu \mathrm{m}$ novolac film, and that these hypotheses are inadequate to explain surface inhibition. Several other theories are explored, including oxidation of the surface, surface roughness effects, etc. The critical ionization dissolution model may offer an explanation for why surface inhibition is observed in novolac, but typically not in poly(p-hydroxystyrene).
\end{abstract}

Keywords: surface inhibition, residual casting solvent, surface roughness, critical ionization dissolution model

\section{INTRODUCTION}

The semiconductor industry's continuing goal of reduction in microelectronic feature sizes requires constant improvements to the microlithographic process. In recent times, many examples can be cited where modeling has played an important role in improving this process. However, in order to simulate the lithographic process, it is necessary to have a fundamental understanding of each processing step. The exposure and latent image transfer are well understood, and can be accurately modeled given the correct parameters. ${ }^{1,2}$ However, the fundamental mechanism for resist dissolution is still widely discussed in the literature. ${ }^{3,4,5}$ In particular it is poorly understood why surface inhibition is observed in many nonchemically amplified resist systems, while the phenomenon is generally absent from chemically amplified (CA) resists. Surface inhibition (or surface induction) describes a slow dissolution rate at the beginning of aqueous base development, which accelerates to a constant, bulk dissolution rate. This changing dissolution rate throughout thickness can be beneficial to the quality of the final resist image. It has been reported that surface inhibition improves contrast by reducing the dissolution rate in unexposed regions of the resist (dark loss), ${ }^{6}$ and this result is confirmed through simulation. Knowledge of the fundamental mechanism of surface inhibition would be beneficial to the rational design and processing of resists - if the dissolution rate versus thickness could be predesigned into the resist, more absorbing resists that would otherwise have sidewall angles below $90^{\circ}$ could be engineered to form more rectangular profiles. It is also beneficial to rigorous modeling of the dissolution process, and it would provide a more complete understanding for the mechanism of aqueous base dissolution. In order to determine the fundamental mechanism of surface inhibition, it is necessary to study the novolac materials in which it is commonly observed.

Several processing conditions affect the extent of inhibition, most notably the bake temperature and time, and of course the resist material. Moreau provides a good summary of the effect of compositional and processing parameters on surface inhibition, and processing "tricks" that can be used to induce the effect. ${ }^{6}$ In this work, the focus is on a fundamental understanding of surface inhibition in a novolac resin. As Dammel points out ${ }^{5}$, surface inhibition in a pure novolac resin is not well-understood, while there are several known mechanisms for surface inhibition in photoresists. For example, Ttopping is observed in chemically amplified resists due to environmental base contamination. While T-topping is a form of surface inhibition, phenomena of this nature that have a well understood mechanism are not considered in this work.

Several theories have been offered in the literature to explain surface inhibition in a novolac film. These hypotheses can be separated into two main groups: The first category suggests a concentration gradient of some photoresist component. The resist film is thought to have a different composition near the surface that would explain the varying dissolution rate 
throughout thickness. The second group of theories propose no such concentration gradient and are derived from the chemistry of the materials and/or fundamental theories of dissolution.

Of the first group, a popular theory (theory \#1) is that a concentration gradient of residual casting solvent exists after spin coating and baking. ${ }^{6,7}$ Several researchers have shown that bulk dissolution rates are dependent upon the residual solvent concentration ${ }^{8,9}$ so it follows that a concentration gradient in residual casting solvent would result in a nonlinear dissolution rate. Beauchemin et. al. ${ }^{7}$ have used attenuated total reflectance Fourier Transform Infrared Spectroscopy (ATRFTIR) to measure solvent gradients, and report a depletion of ethyl lactate (EL) and propylene glycol methyl ether acetate (PGMEA) at the surface of novolac and xylenol resists.

A similar hypothesis for surface inhibition (theory \#2) is that a concentration gradient of photoactive compound (PAC) exists, and is responsible for the nonlinear dissolution rate. This is by far the weakest theory for surface inhibition, since surface inhibition is known to occur in pure novolac resin with no added PAC. ${ }^{10}$ Also, if PAC segregated to the surface causing surface inhibition in unexposed areas, heavily exposed areas would inevitably show surface enhancement. To the knowledge of the authors, that effect has never been observed. In order to confirm that the PAC gradient is indeed an incorrect hypothesis, the concentration gradient of a particular PAC was also measured in this work.

Theory \#3 involves non-linear densification of the resist during the post apply bake (PAB). ${ }^{11}$ It is well known that as solvent is baked off during the PAB, densification of the film occurs. It has been suggested that the film densifies to a greater extent near the surface where the solvent is escaping. A thicker "skin" is thought to form on the top layer of the resist. Dissolution of the film's surface is not slower if considered on a mass per time basis, but if measured as thickness per time, the large concentration of mass at the top of the film appears to dissolve slower than the bulk. ${ }^{11}$

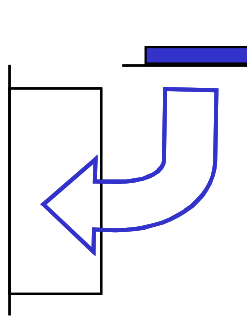

1..

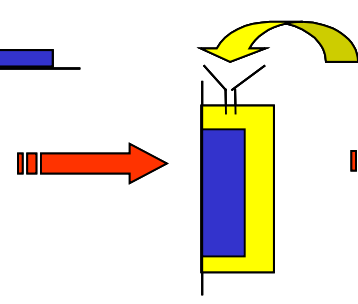

2.

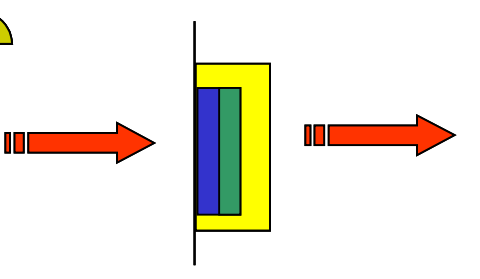

3.
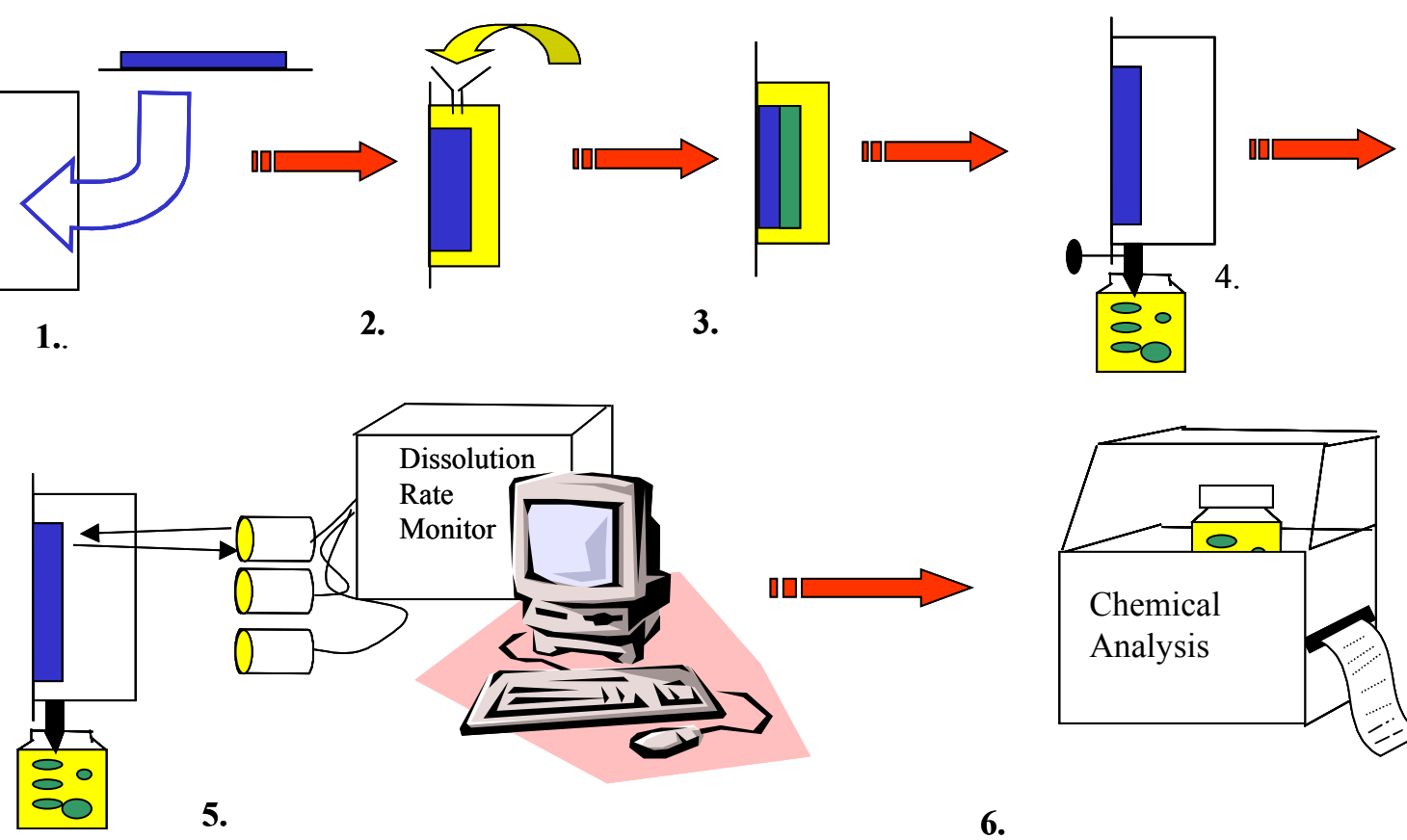

Figure 1. Diagram of Halt Development Technique. 1) A coated wafer is loaded into the cell. 2) Dilute developer is poured into the cell. 3) The resist is allowed to partially dissolve. 4) The dissolved resist is drained from the cell. 5) The intermediate thickness is determined. 6) The top layer is analyzed. The process is repeated for the bottom layer of resist.

Another theory (\#4) is that low molecular weight (MW) polymer chains migrate away from the non-polar air-resist interface due to their polar endgroups. ${ }^{12}$ In film blends of polystyrene and deuterated polystyrene, it has been observed that preferential surface migration can occur based on relative molecular weights. ${ }^{32,33}$ The resist dissolution rate has been shown to be highly dependent upon the polymer MW, ${ }^{13,14}$ so any inhomogeneity in the MW distribution throughout thickness would result in a nonlinear dissolution rate. Note that all of the above theories suggest that the resist has a different composition near the surface of the resist.

To test the first four hypotheses, it was necessary to determine the resist composition at various thicknesses throughout the film. To accomplish this task, a technique termed the "halt development" (HD) technique has been devised 
and is used to separate individual layers of the photoresist film. Using either radio-labeling techniques or the inherent UV-vis absorbance characteristics of a particular resist component, it was possible to analyze chemically individual layers of a resist. In order to test theory \#4, it was necessary to radio-label novolac polymer and separate the polymer into monodisperse samples of varying MW. In a previous work, radio-labeled casting solvent was used in conjunction with the HD technique to determine the distribution of propylene glycol methyl ether acetate (PGMEA) and methoxyethyl ether (diglyme) in a novolac film. The distribution was found to be homogeneous throughout thickness over a range of bake temperatures of $70-110^{\circ} \mathrm{C}^{15}$ The results corresponded well with a PAB model developed by Mack. ${ }^{16}$ The model predicts a concentration gradient of solvent throughout thickness, but one so steep (within the first $5 \%$ of the film) that it is difficult to detect experimentally. The conclusion was that the residual solvent concentration gradients are not responsible for the surface inhibition observed in the novolac used in this work.

Four additional hypotheses are prevalent in the literature and will be discussed in this work. (Note that none of the following theories involve a concentration gradient of any resist component). The first (theory \#5) is that the surface of the resist is oxidized during the $\mathrm{PAB}$. In the presence of heat or UV light, oxygen is known to cause crosslinking of the methylene bridges in ortho-ortho novolac. The reaction is reported by Dammel ${ }^{5}$ and Moreau and is shown in Figure 4. A tacit assumption of this theory is that oxygen is more highly concentrated at the surface of the resist, resulting in a higher $\mathrm{MW}$ at the surface of the resist as the polymer chains undergo crosslinking. Bowden et $a l^{10}$ report that for N64C novolac resin, considerable surface inhibition is observed with a PAB open to air, whereas a PAB under vacuum resulted in very little surface inhibition and faster bulk dissolution rates. An IR study confirmed the growth of the carbonyl peak (indicating crosslinking) in the open air bake, whereas crosslinking was not observed during the vacuum bake. In their resist, slower dissolution rates and a longer induction period are clearly linked to the presence of oxygen during the PAB. ${ }^{10}$ Empirical evidence for the oxidation theory is that the bake temperature has a large effect on the shape and extent of surface inhibition, a result reported by many researchers. ${ }^{10,17,27}$ In this work, the oxidation theory was examined by confirming the effect of bake temperature on dissolution rate and estimating the sorption/concentration gradient of oxygen at various bake temperatures. The FTIR and vacuum bake experiments performed by Bowden et al were also employed.

The next hypothesis (theory \#6) is that the dissolution rate is strongly dependent on the surface roughness, and that the surface roughness increases as dissolution proceeds. The origin of this theory is dissolution simulations that predict surface inhibition based upon an increase in surface roughness. ${ }^{18,19}$ The underlying mechanism is based on the fact that as the surface area available to the developer increases (due to increasing roughness), the dissolution rate increases. Reynolds and Taylor have shown that for APEX-E, the surface roughness does increase as dissolution proceeds with the same functional form observed for the dissolution rate in novolac materials. ${ }^{20}$ The shape of the roughness plot strongly suggests a link to surface inhibition. However, the dissolution rate as a function of thickness has not been previously compared with the surface roughness as a function of thickness. In this work, that direct comparison is made for a novolac polymer film and a poly(p-hydroxystyrene) (PHOST) film.

An interesting explanation for surface inhibition (theory \#7) is discussed by Dammel. ${ }^{5}$ He notes that for tank (immersion) development systems, it has been found that the first few wafers dissolved have slightly slower dissolution rates. The implication is that some dissolved novolac contaminates the developer, functioning as a surfactant that acts as an autodissolution enhancer. Novolac is often added to developer to keep processing conditions constant over time. This idea may explain surface inhibition because, at the beginning of dissolution, the concentration of dissolved novolac in the developer is low. As dissolution proceeds, the concentration of dissolved novolac in the developer near the resist increases, and may act as a surfactant that increases the dissolution rate for the bulk of the film.

One theory that is not directly examined in this work (theory \#8) stems from the "gel layer" theory for dissolution. The assumption of this theory is that base transport into the film is the rate-limiting step for dissolution. The "gel" is a phase boundary between the developer and the bulk of the film with an intermediate composition. Peppas et al discuss this theory for the general dissoluton of glassy polymers. ${ }^{34}$ This theory has been studied extensively as the percolation model for phenolic polymer dissolution by Reiser ${ }^{3}$, and is also discussed in detail by Dammel. ${ }^{5}$ Arcus demonstrated with an interferogram that for a phenolic polymer of high $\mathrm{MW}$, a gel layer was present during dissolution. ${ }^{21}$ However, for novolac films of low MW, the gel layer is either nonexistent or too thin to be observed with interferometry. A recent modeling effort reported that the thickness of the gel layer was on the order of $\sim 2 \mathrm{~nm} .{ }^{22}$ The relationship of this theory to surface inhibition is thought to be as follows: At the beginning of development, no gel layer exists and the dissolution is slow and accelerates as the gel layer forms. The gel layer eventually reaches a constant thickness and the dissolution rate reaches a steady state value. The inhibition period is thought to correspond with the formation of the gel layer.

A contrasting fundamental dissolution theory is the critical ionization (CI) model proposed by our research group. ${ }^{4}$ The assumption of this theory is that the deprotonation reaction is the rate-limiting step for dissolution. The premise is that a critical fraction of ionized sites must be reached in order for an individual chain to dissolve. This model has been quite successful at explaining the MW effect on dissolution rate as well as the anomalous effect of added salts to the developer. ${ }^{4} \mathrm{~A}$ molecular level simulation algorithm in which individual polymer chains are placed on a lattice has been designed. ${ }^{23} \mathrm{~A}$ 
developer front is allowed to ionize a certain fraction of surface sites (monomer units). The chemical structure (pKa) of the polymer has a strong effect on the fraction of surface sites that may ionize. ${ }^{24}$ For example, PHOST has a much lower pKa than novolac (for $\mathrm{pH}$ conditions above 7), and the fraction of surface sites that may ionize on a PHOST film is thus much higher than novolac. In a previous work, the CI model was shown to correctly predict that PHOST dissolves faster than novolac (all relevant parameters being equal). ${ }^{24}$ In this work, the same argument is used to demonstrate that the model also predicts surface inhibition in materials with a low fraction of ionized surface sites (such as novolac). The model also predicts an increase in surface inhibition with decreasing void fraction. The observed surface inhibition in the simulations is a combination of $\mathrm{pKa}$ and roughness effects.

\section{MATERIALS}

The resins and photoresist systems used for this work were novolac, novolac/diazonaphthoquinone, and PHOST. Cresol novolac from Schenectady International ( $\mathrm{MW}=9370, \mathrm{Pd}=4.9)$ and a bis-(1-oxo-2-diazonaphthoquinone sulfonate) (DNQ) from IBM were used. PHOST $(\mathrm{Mn}=8770, \mathrm{Pd}=1.11)$ was obtained from Nippon Soda. The casting solvents used were PGMEA (propylene glycol methyl ether acetate-carbonyl- $\left.{ }^{14} \mathrm{C}\right)$ and diglyme (bis $\left(2-{ }^{14} \mathrm{C}-\right.$ methoxy ethyl) ether). The resists consisted of $77 \mathrm{wt} \%$ solvent and $12 \%$ PAC relative to the solids (in the experiments in which PAC was used). It was not necessary to expose the resist in any experiments. The developer used was AZ 300 MIF tetramethylammonium hydroxide from Clariant Corp. The developer was diluted with deionized water, in a ratio appropiate for the particular experiment.

\section{EXPERIMENTAL AND DISCUSSION}

\subsection{The Halt Development Technique}

The HD technique is described in Figure 1. The general procedure for these experiments was as follows. A coated wafer was placed into the HD cell, and the cell was assembled. An appropriate developer solution was poured into the cell, and the film began to dissolve into solution. After a given amount of time, the cell was drained into a vial and dilute developer was poured into the cell to stop development and wash the cell. The cell was drained into the same vial and the top layer of the resist was successfully separated from the bottom. The procedure was then repeated to dissolve the bottom layer of the resist. The vials were analyzed separately for the resist component of interest. The intermediate thickness of the film was determined by multiwavelength interferometry. Note that only one intermediate thickness was used per film, minimizing unwanted transport ("leaching") of resist components from the bottom layer into the washings, and easing the determination of an intermediate thickness. Thus, several identical films were used to determine the concentration gradient over the entire film. Experiments have been performed to optimize the number of washings and to quantify the extent of leaching. These experiments along with the HD technique are described in more detail elsewhere. ${ }^{15,25}$ The resolution of the technique is estimated to be $30 \mathrm{~nm}$. The estimate is based upon an error estimate for several steps of the process (the uniformity of the thickness, material lost during washing, leaching of material between each layer, etc.) and combining each error into a total error for the thickness. The films used for the HD experiments were $1.2-1.5 \mu \mathrm{m}$ thick, thus a resolution of $30 \mathrm{~nm}$ corresponds to successfully separating layers that are $2-2.5 \%$ of the total thickness. Notice in Figures $6 \& 8$ that surface inhibition extends over $250 \mathrm{~nm}$ into the resist (20-25\% of the film).

\subsection{Investigating Density and PAC gradients by Halt Development}

The HD technique was used to determine the concentration gradient of novolac (density) and PAC. In this case no resist components were specifically labeled. Rather, the characteristic UV-Vis absorbance of both the novolac $(298 \mathrm{~nm})$ and PAC $(343 \mathrm{~nm})$ was measured with a Hewlett Packard 8452A UV-Vis spectrophotometer to determine a concentration at specific depths throughout the film. Concentration standards were prepared of novolac and PAC, and their UV-Vis absorbance spectra were measured to verify that Beer's Law was applicable in the concentration region of interest. Films of Novolac/PGMEA/PAC were spun at $2500 \mathrm{rpm}$ for $30 \mathrm{~s}$ to achieve a film thickness of $\sim 1.2 \mu \mathrm{m}$. The PAB was $90^{\circ} \mathrm{C}$ for $90 \mathrm{~s}$. The films were not exposed. The HD technique was used to obtain specific fractions of the film in solution. Relative concentrations were determined at a specific thickness by normalizing the absorbance at the proper wavelength to the absorbance of a completely dissolved film. The results of this experiment are shown in Figure 2.

The concentration of novolac (density) and PAC were found to be constant throughout thickness within the resolution of the experiment. Thus, the film did not preferentially densify near the surface during the post apply bake ( $\mathrm{PAB}$ ), and the photoactive compound did not preferentially segregate towards the surface. Furthermore, if there existed a concentration gradient in residual solvent or density, one would expect a gradient in the index of refraction over thickness. Ficner et al studied index of refraction gradients in AZ novolac resists with a Metricon Prism coupler. ${ }^{26}$ They observed 


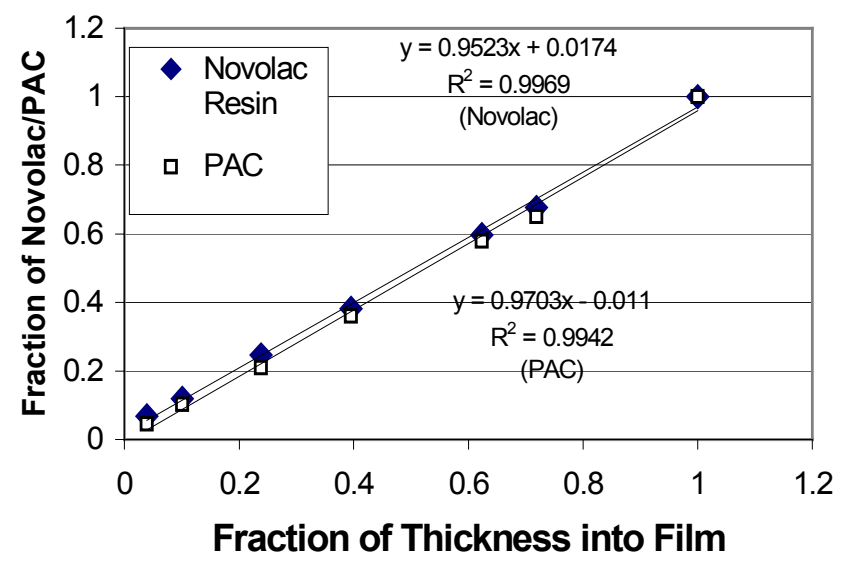

Figure 2. Overall polymer and PAC concentration throughout thickness in a novolac film.

gradients in the index of refraction for thick films $(32 \mu \mathrm{m})$, indicating a significant residual solvent gradient. But with thinner films $(4-8 \mu \mathrm{m})$, the index gradient was "too small to lead to observable bent light modes", and any index gradient was not detectable, which is consistent with our results. Based on these results, the solvent, density, and PAC gradient theories are not viable explanations for surface inhibition for this novolac resin.

\subsection{Investigating Low Molecular Weight Concentration Gradients by Halt Development}

Radio-labeled novolac was synthesized from metacresol and carbon-14 labeled formaldehyde. The details of the synthesis are described elsewhere. ${ }^{12}$ The separation of narrow MW fractions was accomplished by supercritical fluid fractionation at Phasex Corp. The details of this separation and analysis are described elsewhere. ${ }^{12,25}$ The two samples of low molecular novolac "tracers" used to dope the resist had an $\mathrm{Mn}=2130$ and $\mathrm{Pd}=1.26\left({ }^{14} \mathrm{C}\right.$-Tracer $\left.\mathrm{A}\right)$, and the second tracer was over $70 \%$ dimers and trimers $\left({ }^{14} \mathrm{C}\right.$-Tracer B). A Beckman 1801 liquid scintillation counter was used to measure the activity of each sample. Fisher Scintiverse II was the scintillation cocktail used for the developer/resist solutions collected from the HD cell. The H-number quenching calibration for both the labeled solvents and labeled polymer is described elsewhere. $^{12}$ A small amount of each tracer (less than $7 \mathrm{wt} \%$ ) was added to a separate novolac/PGMEA/PAC resist formulation. The spin speeds were adjusted to obtain approximately $1.5 \mu \mathrm{m}$ films. The PAB was $90^{\circ} \mathrm{C}$ for $90 \mathrm{~s}$. The $\mathrm{HD}$ technique was then applied with the protocol discussed previously ${ }^{15}$ to determine the concentration versus thickness of the radio-labeled low MW species.

The results of this experiment are shown in Figure 3. The distribution of the low MW species (both tracers) are homogeneous throughout the thickness of the resist. Note the slightly positive $y$-intercept, indicating a higher than average concentration at the surface. If theory \#4 were correct, there should be should be a depletion of low MW chains (the labeled species) near the surface of the resist. It appears that while the migration theory is plausible, the timescale necessary for this migration to occur is not present in the experiment or in a typical lithographic process.

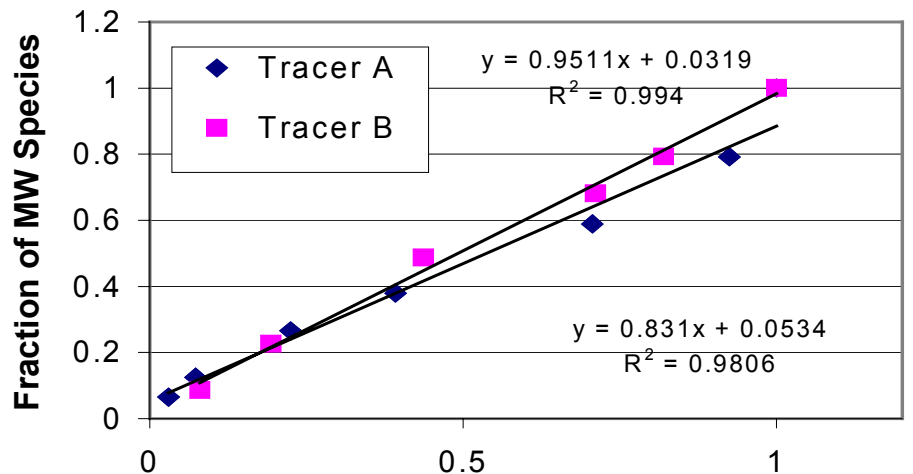

Fraction of Thickness into Film
Figure 3. Low MW concentration throughout thickness in a novolac film 
There also exists some empirical evidence to suggest that MW chain migration is not occurring. For example, a 1.0 $\mu \mathrm{m}$ film consisting of only the monodisperse novolac of $\mathrm{Mn}=2130, \mathrm{Pd}=1.26$, cast from PGMEA and baked at $150^{\circ} \mathrm{C}$ shows a considerable amount of surface inhibition. A blend of this resin and the polydisperse novolac $(\mathrm{Mn}=9330, \mathrm{Pd}=4.9)$ showed no increase in surface inhibition, but a general decrease in the bulk dissolution rate - the expected result if the low MW chains were dispersed evenly throughout the film. If the low MW chain migration theory were correct, one would expect surface inhibition to be strongly dependent on the polydispersity, decreasing considerably at values of Pd close to 1.0 . In our experiments, the extent of surface inhibition did not depend on polydispersity. Moreau reports that using higher MW narrow-dispersity novolac resin is a method of increasing the extent of surface inhibition, ${ }^{6}$ although Dammel presents some data that show increasing surface inhibition with increasing polydispersity. ${ }^{5}$ In conclusion, our experiments provide both direct and indirect evidence suggesting that the migration of low MW chains away from the film surface is not an adequate explanation for surface inhibition in this novolac resin.

\subsection{Oxidation Effects During PAB}

The cross-linking reaction caused by heat (or light) and oxygen and reported by Moreau ${ }^{6}$ and Dammel $^{5}$ is reproduced in Figure 4. This reaction has been studied in the context of understanding the post develop bake used to harden novolac resist features. In the presence of oxygen, chain scission is thought to occur at the methylene bridges followed by cross-linking to a nearby chain. One method of detecting the reaction is by monitoring the growth of the carbonyl peak by Fourier Transform Infrared (FTIR) spectroscopy. Dammel and Moreau point out that this reaction has been observed in an atmosphere with no oxygen, suggesting that oxygen was previously absorbed into the film or "self-oxidation" of the novolac occurred. ${ }^{5,6}$ The oxidation theory for surface inhibition was first suggested due to the strong influence of PAB temperature upon the extent of surface inhibition. The dissolution rate of the novolac used in this study is shown over a range of PAB temperatures $\left(60-150^{\circ} \mathrm{C}\right)$ in Figure 5. (The initial film thickness varied with $\mathrm{PAB}$ temperature. To avoid confusion, the initial film thickness has been normalized to the average thickness of $1.25 \mu \mathrm{m}$ for each experiment). The bulk dissolution rate slows as the temperature is increased, due to the known decrease in residual casting solvent. ${ }^{8,9}$ There is also a dramatic change in the extent of surface inhibition. However, the HD experiments, ${ }^{15}$ the model by Mack ${ }^{16}$ and the results of Ficner ${ }^{26}$ suggest that the change in surface inhibition is not related to a residual solvent concentration gradient in the film. Therefore the oxidation theory deserves close examination for the novolac resin studied in this work.<smiles>Cc1cc(C)c(O)c(Cc2cc(C)cc(C)c2O)c1</smiles><smiles>CCc1cc(C)cc(Cc2cc(C)cc(CC)c2O)c1O</smiles><smiles>Cc1cc(C)c(O)c(C(=O)O)c1</smiles><smiles>C=C1C=CC(=O)C(=CC)C1</smiles><smiles>C=c1ccc(=O)c(=CC)cc1</smiles>

Figure 4. Oxidation and crosslinking reaction of orthoortho novolac at high temperatures or under UV light. ${ }^{5,6}$ 


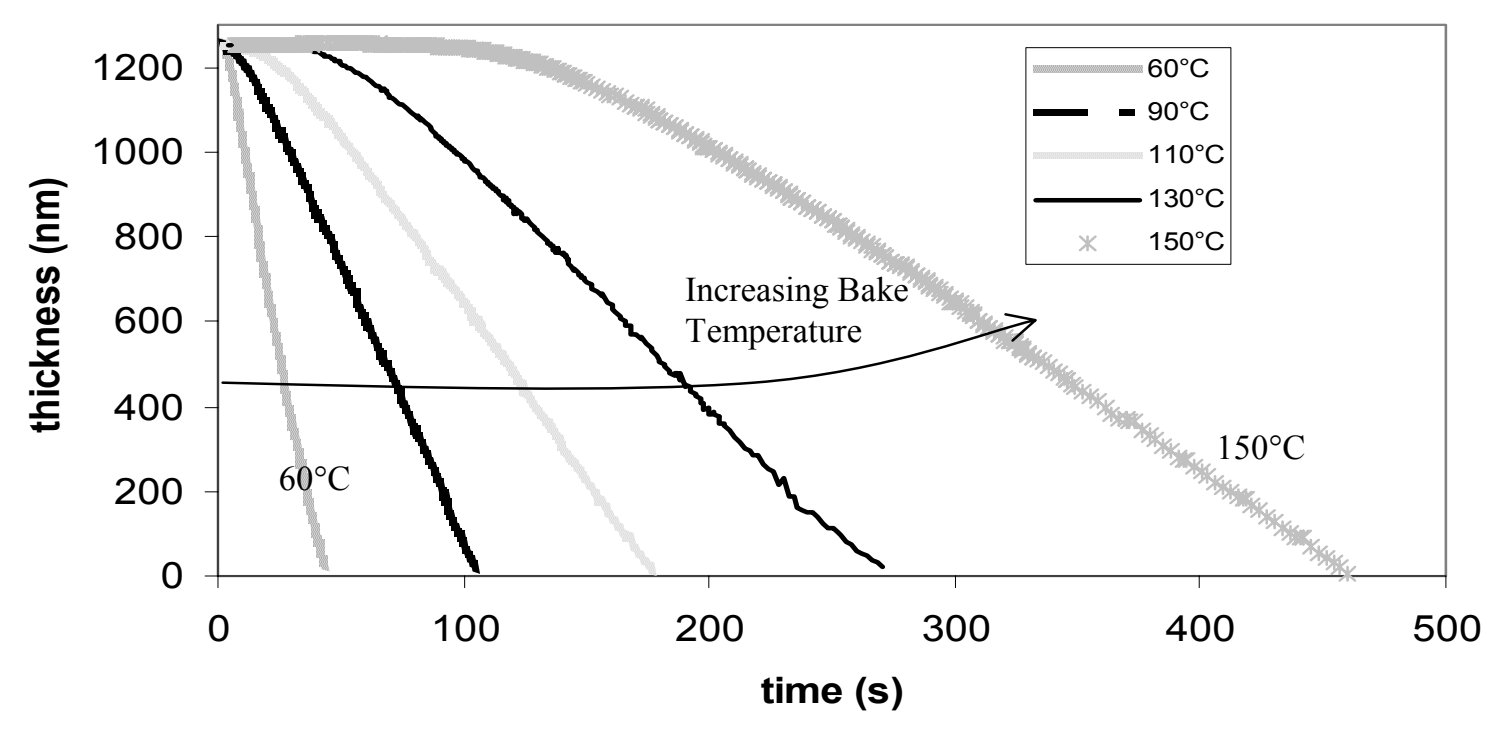

Figure 5. Response of Dissolution Rate to PAB Temperature for Novolac Resin Coated from PGMEA.

One approach is to perform the PAB in an oxygen free environment. This was done by purging the film with industrial grade nitrogen (purchased from Prax Air) at a high flow rate $(\sim 30 \mathrm{ml} / \mathrm{s})$ at several temperatures. Novolac films were cast from PGMEA and spun at $2500 \mathrm{rpm}$ for $30 \mathrm{~s}$ to achieve a film thickness of $\sim 1.2 \mu \mathrm{m}$ depending on the PAB temperature. A developer concentration of $55 \%$ AZ300 MIF and $45 \%$ deionized water was used. A representative result is shown in Figure 6. The dissolution rate versus thickness is plotted for five experiments. All were baked at $150^{\circ} \mathrm{C}$ for $90 \mathrm{~s}$. Three were baked under a nitrogen purge, while two were not. There is a slight decrease in the dissolution rate for the films baked in an oxygen atmosphere, suggesting a small amount of cross-linking (due to oxidation) may be occurring. However, the extent of surface inhibition is the same for all films, suggesting that the cross- linking is not occurring preferentially at the surface, but rather uniformly throughout the bulk of the film.

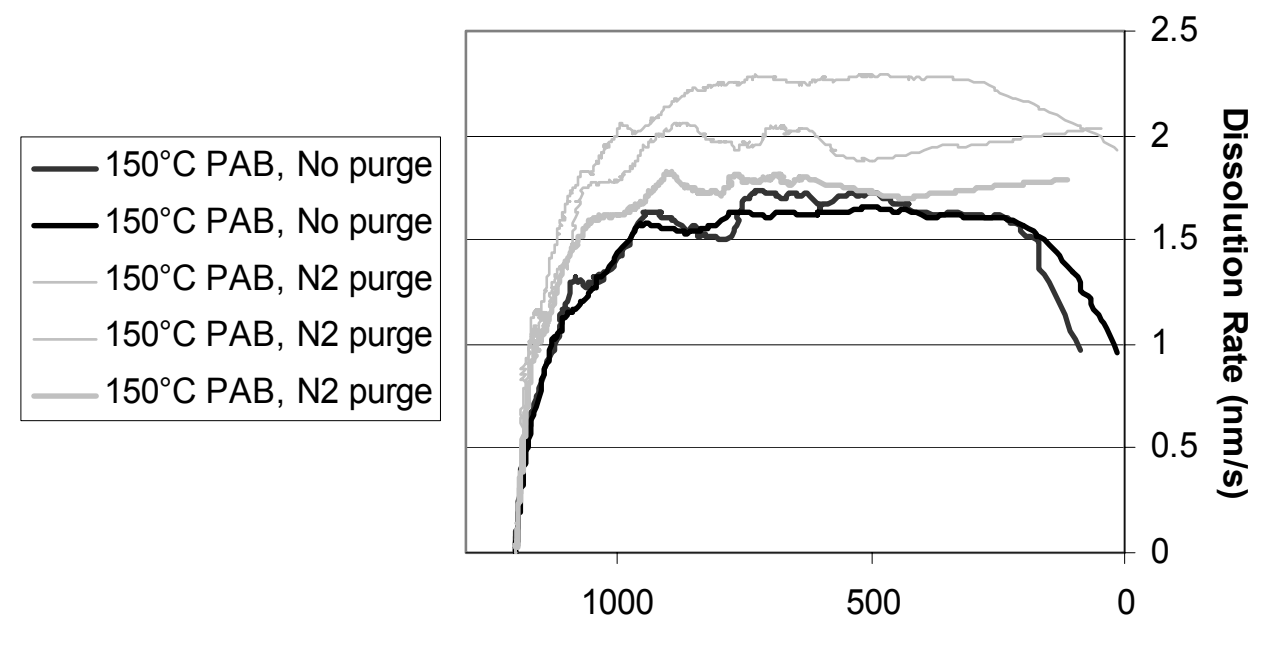

Film Thickness (mm)
Figure 6. Dissolution rate versus film thickness of novolac with and without a nitrogen purge during a $150^{\circ} \mathrm{C} \mathrm{PAB}$. 
FTIR spectra were taken for the novolac films baked over a range of times and temperatures $\left(50-170^{\circ} \mathrm{C}\right)$. The films were cast from diglyme so that residual casting solvent would not contribute to the carbonyl peak. There was no observable increase in the carbonyl region $\left(1550-1700 \mathrm{~cm}^{-1}\right)$ until the resin was baked for 30 minutes at $170^{\circ} \mathrm{C}$. (But note the strong change in surface inhibition at moderate bake temperatures $\left(90^{\circ} \mathrm{C}\right)$ ).

However, only a few cross-linking events are needed in order to double locally the MW of the resin and drastically affect the dissolution properties - possibly few enough events that the carbonyl band would not show a large increase. Also, oxygen may be absorbed during the spin coating of the polymer resin, which may account for observed surface inhibition even during a PAB purged with nitrogen. The main issue then is the sorption behavior of oxygen into the film during spin coating and baking. A tacit assumption of the oxidation theory is that a concentration gradient of oxygen is present throughout the bulk of the film that would lead to a concentration gradient in MW as the cross-linking reaction occurs.

The mass transfer problem of sorption of small penetrant molecules into a flat slab from one side is a trivial one if Fickian diffusion is assumed and diffusivity data is known. ${ }^{28}$ Diffusion of oxygen through many polymer membranes has been well studied, and a reasonable estimate can be made of the diffusivity of oxygen through novolac. The diffusivity of oxygen through a variety of polymer membranes is reported to be between $3.3 \times 10^{-8}$ and $5.5 \times 10^{-6} \mathrm{~cm}^{2} / \mathrm{s}$ at $25^{\circ} \mathrm{C}^{28,29}$ Assuming that the concentration of oxygen is low in the resist formulation, the oxygen sorption occurs during room temperature spin coating and the PAB. Figure 7 shows the concentration gradient and time dependence of oxygen sorption into a $1 \mu \mathrm{m}$ film, with the diffusivity of oxygen assumed to be equal to $1 \times 10^{-8} \mathrm{~cm}^{2} / \mathrm{s}$ (on the low side of the values reported for other membrane materials). Within one second, oxygen has permeated nearly the entire film. Also, the diffusivity will increase by $2-3$ orders of magnitude at $150^{\circ} \mathrm{C}$, making oxygen sorption even faster during the PAB. Unless the diffusivity of oxygen through novolac is anomalously low, there does not exist a significant concentration gradient of oxygen during the PAB that could lead to preferential crosslinking at the surface of a novolac film.

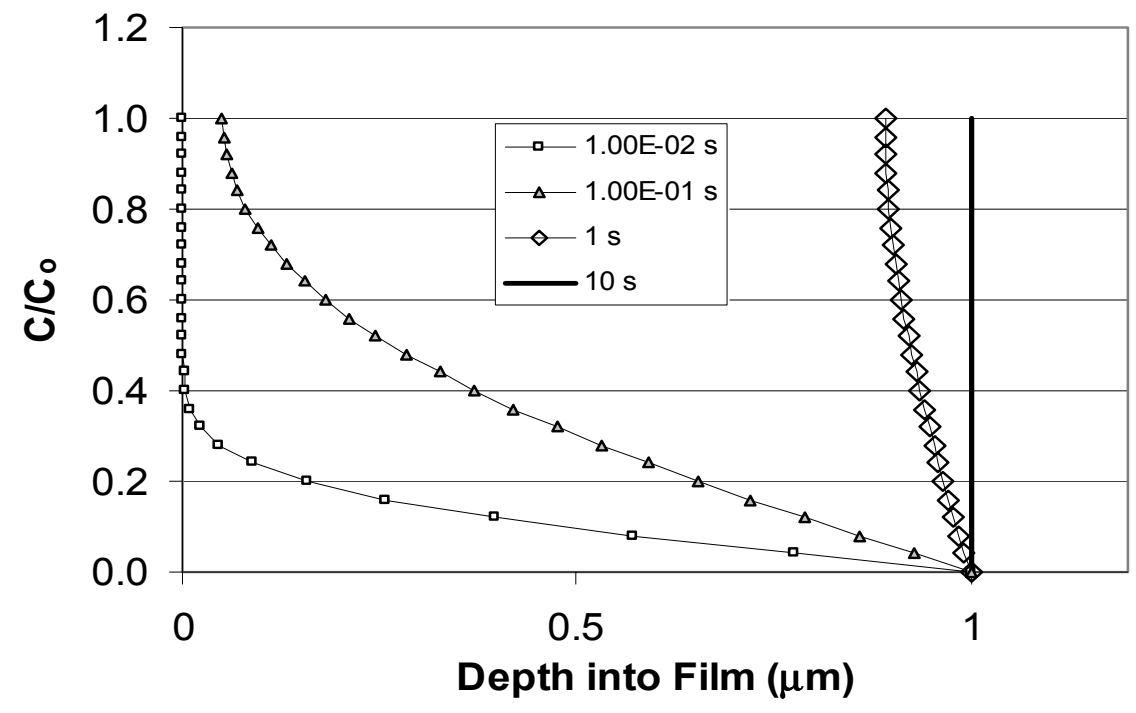

Figure 7. Calculated sorption of oxygen into a thin polymer film. The conc-entration, $\mathrm{C}$, is normalized to the concentration of oxygen at the surface of the film, $\mathrm{C}_{0}$. $\left(\mathrm{D}_{\text {oxygen }}=1 \times 10^{-8}\right.$ $\left.\mathrm{cm}^{2} / \mathrm{s}\right)$

\subsection{Novolac Auto-Dissolution Enhancement}

The premise of the dissolution-enhancement theory is that dissolved novolac near the undissolved film acts as a dissolution promoter. ${ }^{5}$ (For the time being, the exact mechanism of this enhancement is not a point of concern). The simplest method for determining if dissolved novolac alters the dissolution rate (for this novolac) is to perform the immersion-tank experiment that was the origin of the theory. The experiment involves adding novolac to the developer prior to dissolution, and observe the effect on the bulk dissolution rate. (Note that the experiment performed in this case was done in puddle development mode, not with an immersion tank.) 


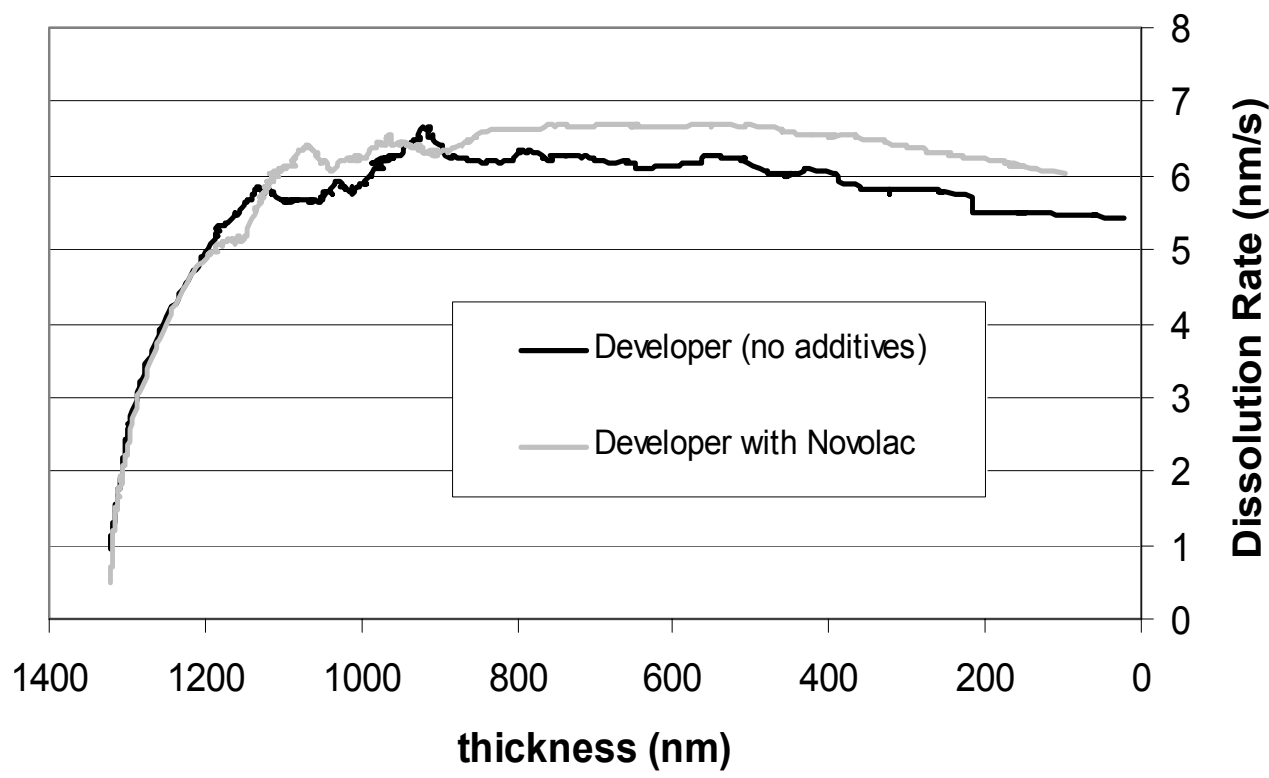

Figure 8. Dissolution rate versus thickness of a novolac film with and without adding novolac to the developer.

The developer solution used was 55:45 AZ300MIF to deionized water (by volume). Aliquots of the developer were chosen and novolac was added in various ratios to the developer, up to $1 \%$ by weight. The novolac/developer solutions were stirred for up to an hour. At higher concentrations, not all of the added novolac dissolved. Films of novolac were cast from PGMEA at a spin speed of $2500 \mathrm{rpm}$ for $30 \mathrm{~s}$ with a PAB of $90^{\circ} \mathrm{C}$ for $90 \mathrm{~s}$. The films were dissolved with the various developer solutions, and the dissolution was monitored with multiwavelength interferometry. A typical result is shown in Figure 8. No change was observed in the bulk dissolution rate with the various developer solutions. Also, there was no change in the extent of surface inhibition. The novolac used in this work does not enhance or inhibit the bulk dissolution rate when dissolved in the developer. It appears that auto-dissolution enhancement is not an adequate explanation for surface inhibition in the system studied.

\subsection{Surface Roughness Effects During Dissolution}

The roughness experiments were performed using the novolac and PHOST resin coated from PGMEA. The spin speed was adjusted to obtain $\sim 1 \mu \mathrm{m}$ films, which were baked at $90^{\circ} \mathrm{C}$ for $90 \mathrm{~s}$. In this case, the HD technique was used, although it was not necessary to collect the effluent. The development was quenched with water and the films were blown dry with nitrogen. The surface roughness was calculated from images taken on a Park Scientific Instruments Autoprobe AFM. It was assumed that quenching development with water and blow-drying does not significantly alter the surface roughness.

The results of this experiment are shown in Figure 9. The average surface roughness and dissolution rate are plotted versus thickness. For each resin, the roughness trend is similar. Novolac and PHOST were smooth when first spin coated (under $1 \mathrm{~nm}$ of roughness), but as dissolution proceeded the roughness increased, reaching a bulk value of 2-3 nm. The roughness behavior is consistent with similar experiments performed by Reynolds and Taylor on APEX-E, ${ }^{20}$ and is qualitatively consistent with observed roughness behavior from latticed-based simulation. ${ }^{18,19}$

For novolac, there is a correlation between the roughness curve and the dissolution rate. However, for PHOST, there is no correlation between surface roughness and dissolution rate. It is difficult to argue that surface roughness effects alone are responsible for surface inhibition when the same roughness trends are observed in materials with a wide range of dissolution rate profiles. That is, if only roughness effects were responsible for surface inhibition, one would expect to see surface inhibition in PHOST and APEX-E. The CI model offers an explanation for this strange paradox based upon the pKa of each resin material. 

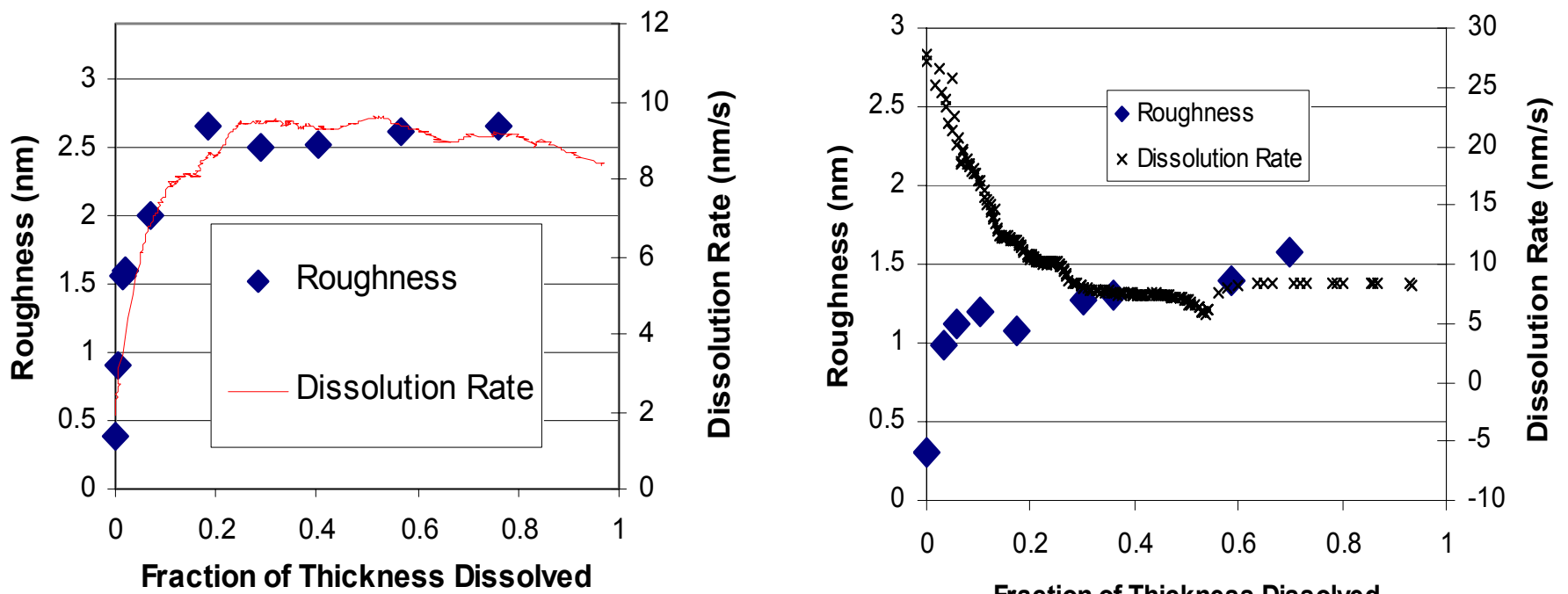

Fraction of Thickness Dissolved

Figure 9. (a) Surface roughness and dissolution rate versus thickness for a novolac resin. (b) Surface roughness and dissolution rate versus thickness for a PHOST resin.

\section{MODELING RESULTS}

The novolac resin studied in this work has been shown to have no significant concentration gradients of residual solvent, low MW chains, PAC or overall density over a $1 \mu \mathrm{m}$ film. Furthermore, there is no evidence of preferential oxidation near the surface, or auto-dissolution enhancement occurring. The increase in roughness during dissolution may partially explain the observed surface inhibition in novolac, but lends no explanation to the dissolution profile of PHOST. Any theory put forth to explain the dissolution behavior of both materials must incorporate the different chemistries of the two materials. (The oxidation theory and the auto-dissolution enhancement theory are candidates, but both have been disproved for the novolac resin of interest.)

The CI model proposed by our research group has provided some insight to the issue. The main input parameters to the model are the fraction of ionized surface sites $(\alpha)$ and the critical fraction of sites necessary to render a polymer chain insoluble $\left(f_{\text {crit }}\right)$. The latter value, $f_{\text {crit }}$, is assumed to change little (if any) from polymer to polymer. However, $\alpha$ is very dependent upon the structure and acidity of a given polymer. Specifically, the equilibrium of the deprotonation reaction dictates that $\alpha$ is related to the $\mathrm{pH}$ of the developer near the surface of the film $\left(\mathrm{pH}_{\mathrm{o}}\right)$ and the $\mathrm{pK}_{\mathrm{a}}$ of the polymer by: ${ }^{24}$

$$
\alpha=\frac{10^{p H_{o}-p K a}}{1+10^{p H_{o}-p K a}}
$$

The acidity function of the polymer dictates that $\alpha$ is related to the $\mathrm{pKa}$ of the film ${ }^{24}$ :

$$
\mathrm{pK}_{\mathrm{a}}=f(\alpha)
$$

Where Equation (2) is the acidity function of the polymer. Recently ${ }^{30}$, our modeling efforts have included a third effect,

$$
\mathrm{pH}_{\mathrm{o}}=f\left(\mathrm{pH}_{\text {bulk }}, \alpha\right)
$$

in which the surface $\mathrm{pH}$ of the developer $\left(\mathrm{pH}_{\mathrm{o}}\right)$ is lower than that of the bulk due to electrostatic repulsion of the negative hydroxide ions by the overall negative surface charge of the dissolving polymer (formation of an electric double layer). The solution to the Poisson-Boltzmann equation to determine Equation (3) and the calculation of $\alpha$ (for both novolac and PHOST) is reported elsewhere. ${ }^{30}$ The calculations are based on a similar problems discussed by Israelachvili. ${ }^{31}$ The main assumption of this third effect is that the resist is impenetrable to hydroxide ions. While this is already a tacit assumption of 
the CI model (rather, the film dissolves before the hydroxide ions penetrate), for now the effect of surface forces is included to place a lower bound on the value of $\alpha$ for a given polymer. The values of $\alpha$ reported are for a developer concentration of $0.26 \mathrm{~N}$. For PHOST, the calculated value of $\alpha$ is between 0.8-0.99. For novolac, the calculated value of $\alpha$ is in the range 0.5-0.8. The overall result is that $\alpha$ is considerably lower for novolac than for PHOST. This result was used to explain why PHOST dissolved much quicker than novolac, all relevant conditions being equal. ${ }^{24}$ Here, this result is used to provide insight into surface inhibition.

A new addition to the CI model has been ionization equilibrium. Previously, a fraction of surface sites $(\alpha)$ would ionize in a given time step. For an undissolved chain, the same sites remained ionized for the remainder of the simulation (dissolution of the film). However, the equilibrium of the deprotonation reaction is known to be quite fast. The new algorithm assumes that a time step in the simulation is greater than the time needed for equilibrium, and that ionized sites on undissolved chains can reprotonate. The ionized sites are randomly redistributed in each time step while keeping the overall fraction of ionized sites constant. The system is able to sample many more configurations, and also follows the principle of microscopic reversibility. At high values of $\alpha\left(\alpha>f_{\text {crit }}\right)$, this added change has little effect on the simulations. However, at low values of $\alpha\left(\alpha \sim f_{\text {crit }}\right)$, the effect is quite significant. Previously, when $\alpha$ approached $f_{\text {crit }}$, the system would often become "stuck" in a state where no chains would dissolve. Now, a new configuration is sampled in each time step, sometimes going through several time steps until the system becomes "unstuck". This effect is important at the surface, where the film starts smooth, and then the roughness increases as dissolution proceeds.

To illustrate, consider a lattice of monodisperse chains of degree of polymerization equal to 30 and a void fraction of 0.2. In this example, $\mathrm{f}_{\text {crit }}$ is set to 0.6 . PHOST and novolac can be examined in the simulation simply by changing the value of $\alpha$. (Larger values of $\alpha$ are correlated with PHOST, while smaller values are correlated with novolac). The thickness versus time in the simulations is shown for a range of $\alpha=0.7-0.9$ in Figure 10(a). First note that the bulk dissolution rate is slower at smaller values of $\alpha$, as would be expected from experiment. It is also evident that higher values of $\alpha$ result in only a slight amount of surface inhibition. Conversely, lower values of $\alpha$ result in a significant amount of surface inhibition. At $\alpha=0.7$, over 300 time steps elapse before a bulk dissolution rate is reached. The total time to clear is just over 800 time steps. Over $1 / 3$ of the dissolution time is during the "inhibited" period at the beginning of dissolution. But, this comparison suggests that surface inhibition is due to a combination of two things. Most importantly, the increased $\mathrm{pK}_{\mathrm{a}}$, (and thus decreased $\alpha$ ) of novolac. However, the increase in roughness during dissolution also plays a big role. If the initial spin coated film started out as rough as a dissolving film, then surface inhibition (as observed in the simulations) would also not be present. Thus, the simulations suggest that surface inhibition is a combination of $\mathrm{pK}_{\mathrm{a}}$ and roughness effects, and provide an explanation for the general observation that novolac films show surface inhibition whereas PHOST films do not.
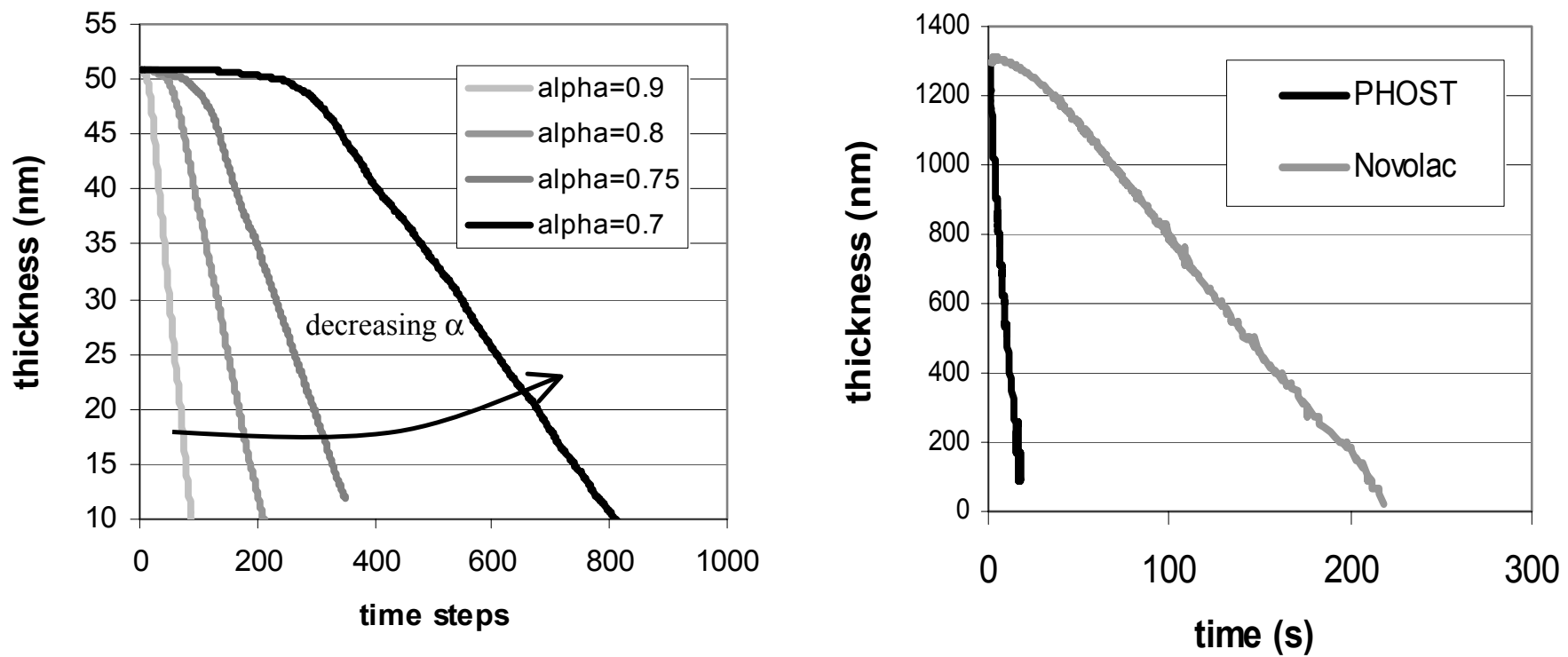

Figure 10 (a) Critical Ionization Dissolution Simulation showing effect of changing the fraction of ionized surface sites, $\alpha$. (cell height is equal to $0.7 \mathrm{~nm})$ (b) Dissolution of PHOST $(\mathrm{Mn}=8770, \mathrm{Pd}=1.1)$ and novolac $(\mathrm{Mn}=9370, \mathrm{Pd}=4.9)$ under identical conditions. 
The simulations were compared to an experiment, shown in Figure 10(b). The dissolution profile of PHOST $(\mathrm{Mn}=8770, \mathrm{Pd}=1.11)$ and novolac $(\mathrm{Mn}=9300, \mathrm{Pd}=4.9)$ were compared. All other formulation parameters, developer concentraton, etc. were kept constant. Qualitatively, the simulations capture the correct trend. The most obvious difference is that the surface inhibition observed in the simulations is always "sharp". That is, the dissolution rate changes abruptly from near zero to the bulk dissolution rate over only a few nanometers. Experimentally, a more curved profile is usually observed over as much as $100 \mathrm{~nm}$. (Figure 10(b)). The difference may be due to mass transfer effects that are not considered in the model.

Another interesting trend emerges from the model. Figure 11 shows a simulation in which the void fraction of the lattice is varied between 5 and $30 \%$. In the lattice model, voids are considered to be either void fraction or residual casting solvent, so a void fraction of $30 \%$ is not an unreasonable value at low PAB temperatures. The other simulation parameters are kept constant at $\mathrm{f}_{\text {crit }}=0.6, \alpha=0.8$, and degree of polymerization of 30 . The decrease in void fraction decreases the initial surface roughness, which has a large effect on the inhibition period. At $30 \%$ void fraction there is almost no surface inhibition, but at $5 \%$ void fraction there is considerable surface inhibition, over a depth of $\sim 8 \mathrm{~nm}$. This is an interesting trend when compared to the change in surface inhibition with PAB temperature shown in Figure 5. At low PAB temperatures, considerable amounts of PGMEA are present in the film. As much as $20 \mathrm{wt} \%$ has been reported to be present after a $70^{\circ} \mathrm{C}$ PAB for 90 seconds. ${ }^{12}$ At higher PAB temperatures, nearly all the solvent is baked off, and the film is annealed, reducing the intrinsic void volume to (on the order of) $3 \%{ }^{26}$ The CI lattice model predicts the roughness to increase from 0.21 to $0.36 \mathrm{~nm}$ over the range of void volume presented. This small increase in roughness is difficult to confirm experimentally. Again, the depth of the inhibition predicted in the model is smaller than observed experimentally. The question of whether the surface inhibition response to $\mathrm{PAB}$ temperature can be explained by variation in roughness and void fraction/residual casting solvent is a topic of future study.

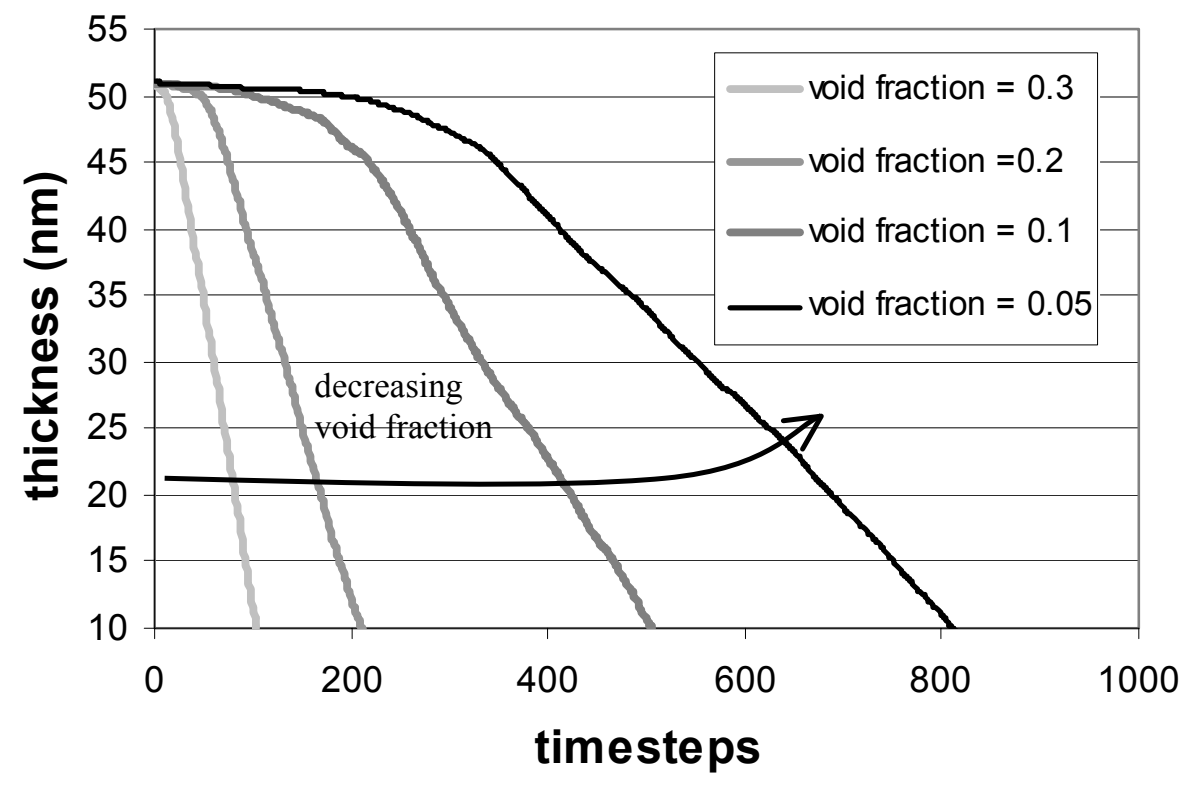

Figure 11. Critical Ionization Dissolution Simulation showing effect of changing the lattice void fraction. $(\mathrm{dp}=30, \alpha=0.8$, $\mathrm{f}_{\text {crit }}=0.6$ )

\section{CONCLUSIONS}

Using the HD technique the novolac resist studied in this work was shown to be homogeneous throughout thickness in residual casting solvent, low MW polymer species, average concentration of polymer, and PAC. The oxidation/crosslinking and auto-dissolution enhancement theories are also inadequate to explain surface inhibition for this resin. The roughness of the novolac resin during dissolution does correlate with dissolution rate, but for PHOST there is absolutely no correlation. However, if the $\mathrm{pKa}$ of each material is considered, the CI model offers an explanation for the presence of surface inhibition in novolac resins and the absence of surface inhibition from PHOST resins. The model also predicts a strong dependence of surface inhibition to the void fraction of the lattice, which may aid in explaining the experimentally observed response of surface inhibition to $\mathrm{PAB}$ temperature. 


\section{ACKNOWLEDGEMENTS}

The authors wish to thank Dr. Ralph Dammel of Clariant, Professor Koros and Professor Bonnecaze (of The Univeristy of Texas at Austin) for insightful discussions and Professor Korgel and Lindsay Pell at The University of Texas at Austin for use and assistance with their AFM. We wish to thank Clariant, Nippon Soda, IBM, and Schenectady International for donation of materials. We would like the thank Chuck Szmanda for discussions about the oxidation effect. We also wish to thank Dr. Hinsberg at IBM for his assistance. The HD cell is a variation of his flow cell design. This work was funded by SRC/DARPA, under contract \# 2000-MJ-752.

\section{REFERENCES}

1. W.G. Oldham,,et al.,IEEE Trans. Electron. Devices., ED-26.4,717,(1979)

2. C.A. Mack, Proc. SPIE, 538,207,(1985)

3. A. Reiser, Z. Yan, Y.K. Han, and M. S. Kim, J. Vac. Sci. Technol. B., 18(3), 1288, (2000)

4. P.C. Tsiartas, L.W. Flanagin, C.L. Henderson, W.D. Hinsberg, I.C. Sanchez, R.T. Bonnecaze, and C.G. Willson, Macromolecules, 30, 4656, (1997)

5. R.R. Dammel, Diazonaphthoquinone-based Resists, SPIE Optical Engineering Press, 1993

6. W.M. Moreau Semiconductor Lithography: Principles, Practices, and Materials, Plenum Press, New York, New York, 1988

7. B.T. Beauchemin Jr. and C.E. Ebersole, Proc. SPIE, 2438, 261, 1995

8. V. Rao, W. D. Hinsberg, C.W. Frank, and R.F.W. Pease Proc. SPIE, 1925, 538, (1993)

9. A.B. Gardiner, A. Qin, C.L. Henderson, S. Pancholi, W.J. Koros, R.R. Dammel, C. Mack, W.D. Hinsberg, C.G. Willson, Proc. SPIE, 3049, 850, (1997)

10. M.Bowden, L. Thomspson, S. Farenholtz, and E. Doerries, J. Electrochem. Soc. 128, 1304 (1981)

11. M.A. Toukhy, Proc. SPIE, 771, 263, (1987)

12. A.B. Gardiner PhD Dissertation, University of Texas at Austin, 1999

13. R.D.Allen et al. Proc. SPIE, 2438, 250, (1995)

14. P.C. Tsiartas, L.L. Simpson, A. Qin, R.D. Allen, V.J. Krukonis, P.M.Wetmore, and C.G. Willson, Proc. SPIE, 2438, 261, (1995)

15. A.B. Gardiner, S.D. Burns, A. Qin, and C. G. Willson, J. Vac. Sci. Technol. B, 19(1), 136, (2001)

16. C. A. Mack, K.E. Mueller, A.B. Gardiner, J.P. Sagan, R.R. Dammel, C.G. Willson, J. Vac. Sci. Technol. B, 16(6), 3779, (1998)

17. F.H. Dill et al. IBM J. Res. Dev., 21, No. 3, 210, (1977)

18. L.W. Flanagin, V.K.Singh, C.Grant Willson, J. Vac. Sci. Technol. B, 17(4), 1371-1379 (1999)

19. P. Trefonas, Proc. SPIE, 1086, 484, (1989)

20. G.W. Reynolds and J. W. Taylor, J. Vac. Sci. Technol. B, 17, 334-344, (1999)

21. R.A. Arcus, Proc. SPIE 631, 124 (1986)

22. J.Y Cho, S.J. Choi, B.U. Kim, J.M. Park, and S.J. Lee, Proc. SPIE, 3999, 432, (2000)

23. L.W. Flanagin, V. K.Singh, and C.G. Willson, J. Poly. Sci., Physics, 37,2103-2113 (1999)

24. L. W. Flanagin, C.L. McAdams, W.D.Hinsberg, I.C. Sanchez, C.G. Willson, Macromolecules, 32(16), 5337-5343 (1999)

25. S.D.Burns, A.B. Gardiner, V.J. Krukonis, P.M. Wetmore,A. Qin, and C.G. Willson, Proc. Amer. Chem. Soc., PMSE, 81, 81-84 (1999)

26. S. Ficner, R.R. Dammel, Y. Perez, A. Gardiner, and C.G. Willson, Proc. SPIE 3049, 838 (1997)

27. F. Dill and J. Shaw, IBM J. Res. Dev. 21, 210 (1977)

28. A.L. Hines and R.N. Maddox, Mass Transfer: Fundamentals and Applications, Prentice Hall, Englewood Cliffs, New Jersey, 1985

29. Encyclopedia of Polymer Science and Engineering, Supplement Volume, Second Edition, John Wiley \& Sons, Inc, 1989

30. S. D. Burns, Surface Phenomenon Class Project (2000):

http://willson.cm.utexas.edu/Research/Sub Files/Surface Phenomena/index.html

31. J. Israelachvili., Intermolecular and Surface Forces, $2^{\text {nd }}$ Ed., Academic Press, 213-243, (1992)

32. P.P. Hong and F.J. Boerio, Macromolecules, 27, 596-605, (1994)

33. A. Harihan et al., J. Chem. Phys., 99(1), 656-663, (1993)

34. B. Narasimhan, and N.A., Peppas, Macromolecules, 29, 3283-3291, (1996) 